\title{
5 Resümee: Ein statisches Metaphernverständnis, geprägt von der Repräsentations-Idee und einem Sender-Empfänger-Modell von Kommunikation
}

Ohne dass dies dezidiert thematisiert oder reflektiert wäre, verhalten sich die in den vorherigen Kapiteln diskutierten Ansätze nun zu einem klassisch gewordenen filmtheoretischen Befund. Denn mit der Entscheidung, sich Metaphern in audiovisuellen Bildern aus Perspektive der Konzeptuellen Metapherntheorie zuzuwenden, positionieren sie sich zu einem für traditionelle Sprachtheorien provokanten Fazit, wie es aus Beschäftigungen mit der Frage, inwiefern Film als Sprache bzw. als etwas Sprachähnliches betrachtet werden kann, gezogen wurde:

Die ersten Filmsemiotiken sind ja auf die Grenze gestoßen, die darin liegt, dass die montierten Bildfolgen des Films wesentliche Bestandteile der Sprache nicht bereithalten: es gibt weder logische noch grammatikalische Strukturen, die eine Sprache des Films konstituierten, und es gibt auch kein Lexikon, mit dem sich filmische Wörter nachschlagen oder übersetzen ließen. [...] Insofern ist jeder Film, oder jede Folge filmischer Bewegungsbilder ein Sprechen [...] ohne Sprache. ${ }^{1}$

Einer solchen Sprach(system)losigkeit audiovisueller Bilder begegnen nun die KMT und die daran orientierten Ansätze, indem sie den Rahmen verschieben. So kommt - eben mit dem maßgeblich metaphorisch strukturierten konzeptuellen System - ein anderes Bezugssystem in den Blick, das indifferent gegenüber medialen Ausdrucksformen ist: Unabhängig davon, ob man es mit sprachlich

1 Gertrud Koch: Latenz und Bewegung im Feld der Kultur. Rahmungen einer performativen Theorie des Films. In: Performativität und Medialität. Hrsg. v. Sybille Krämer. München: Wilhelm Fink 2004, S. 163-188, hier S. 163, Herv. i. O. Vor allem auf Metz' Arbeiten wird sich hier bezogen. Vgl. z. B. Metz: Semiologie des Films; siehe hierzu auch Thomas Elsaesser/Emile Poppe: Film. In: The Encyclopaedia of Language and Linguistics, Bd. 3. Hrsg. v. R.E. Asher. Oxfords: Pergamon Press 1994, S. 1225-1241. Aus dem Umstand, dass filmischen Bildern kein Sprachsystem zugrunde liegt, wird die vorliegende Arbeit freilich nicht den Schluss ziehen, wie er sich aus semiotischer (bzw. semiologischer) Sicht anbietet: dass nämlich jede Bedeutungsgebung filmischer Bilder deshalb auf Codes, d. h. auf Konventionalisierungen basieren würde, wie Koch diese Perspektive resümiert. Vgl. zur Code-Diskussion der Semiologie auch Jörg Schweinitz: Film und Stereotyp. Eine Herausforderung für das Kino und die Filmtheorie. Zur Geschichte eines Mediendiskurses. Berlin: Akademie-Verlag 2006, hier S. 215-223.

Ә Open Access. () 2020 Christina Schmitt, publiziert von De Gruyter. (G) BY-NC-ND Dieses Werk ist lizenziert unter der Creative Commons Attribution-NonCommercial-NoDerivatives 4.0 Lizenz. https://doi.org/10.1515/9783110614619-005 
oder filmisch artikulierten (kreativen bzw. konzeptuellen) Metaphern zu tun hat jedes Mal, so die Annahme, manifestiert sich ein System von Konzepten.

Das impliziert jedoch etwas Entscheidendes. Denn infolgedessen zeigt sich in der KMT die traditionsreiche Repräsentations-Idee. Diese hat sich maßgeblich an der Idee des Zeichens herausgebildet, mit der eine „Ineinssetzung von Zeichen und Bezeichnetem“, wie sie im magischen Denken des Rituals vorherrschte, abgelöst wurde. ${ }^{2}$ Und eben die Trennung des zuvor In-eins-Gesetzten ist, so die Philosophin Sybille Krämer, „der Lebensnerv der Idee der ,Repräsentation““: „Nicht Epiphanie, also Gegenwärtigkeit, vielmehr Stellvertreterschaft, also Vergegenwärtigung, ist das, was die Zeichen für uns zu leisten haben.“3 Damit aber werde die Welt „doppelbödig“:

Etwas, das unseren Sinnen zugänglich ist, wird interpretiert als raum-zeitlich situierte Instanziierung von etwas, das nicht mehr unmittelbar gegeben ist, gleichwohl jedoch der singulären Erscheinung logisch und genealogisch vorausgeht. Mit dem Phänomen des repräsentationalen Zeichens spaltet sich die Welt auf in eine ,Tiefenstruktur', die ein universelles Muster birgt, und eine ,Oberfläche', die dieses Muster unter jeweils konkreten und dabei auch einschränkenden - Umständen aktualisiert. Das, was erscheint, wird zum Derivat von etwas, das hinter der Erscheinung liegt, also unseren Sinnen nicht zugänglich ist. Dieses in der Semiotik der Repräsentation verwurzelte Weltverhältnis sei [...] das „ZweiWelten-Modell“ genannt. ${ }^{4}$

Den Intuitionen dieses Zwei-Welten-Modells folgen, so Krämer, „[n]ahezu alle modernen sprachtheoretischen Ansätze, aber auch weite Bereiche der Texthermeneutik und der Bedeutungstheorien“ - sowie Ansätze in der Kognitiven Linguistik: Krämer führt als Beispiel für einen solchen Ansatz Noam Chomskys Transformationsgrammatik an, in der es ,um das Wissenssystem, das als ein inneres mentales Organ das äußere Sprachverhalten hervorbringt“, geht. ${ }^{5}$ Indem die (von Krämer nicht angesprochene) Konzeptuelle Metapherntheorie nun Erfahrung zum Ausgangspunkt von Bedeutung macht, ist die Kognitive Linguistik Lakoff'scher Prägung zwar als explizite Gegenbewegung zu Chomskys Ansatz eines regelbasierten Systems angelegt; als Gegenbewegung $\mathrm{zu}$ einem System-Modell also (welches ursprünglich weniger auf eine Bedeutungs- denn auf eine Syntaxtheorie zielte), bei dem von einer Modularität des Geistes ausgegangen und die Existenz

2 Sybille Krämer: Sprache - Stimme - Schrift. Sieben Gedanken über Performativität als Medialität. In: Performanz. Zwischen Sprachphilosophie und Kulturwissenschaften. Hrsg. v. Uwe Wirth. Frankfurt a. M.: Suhrkamp 2002, S. 323-346, hier S. 323, Herv. CS.

3 Ebd.

4 Ebd., S. 324.

5 Ebd. Zur Transformationsgrammatik vgl. z. B. Noam Chomsky: Regeln und Repräsentationen [1980]. Frankfurt a. M.: Suhrkamp 1981. 
eines mentalen Lexikons postuliert wird, und bei dem körperliche Erfahrung keine Rolle spielt. ${ }^{6}$ Doch trotz dieser Absetzungsbewegung, die vor allem in den Anfängen der KMT vorherrschte, lässt sich argumentieren, dass auch die KMT am Zwei-Welten-Modell ausgerichtet ist. Zum einen wäre ihre medienindifferente Anlage anzuführen. Im Zwei-Welten-Modell „gehören Medien ausschließlich der Realisierungs- und Instantiierungsebene [sic] an“ und kommen dementsprechend nur dann ins Spiel, „wenn vom medienindifferenten [...] Kenntnissystem unter raum-zeitlich spezifizierbaren Bedingungen Gebrauch gemacht wird“.7 Diesbezüglich sei an die oben dargelegte Position Lakoffs erinnert, demzufolge eine komplexe konzeptuelle Metapher durch ein Set ontologischer Korrespondenzen zweier Konzept-Bereiche begründet ist, was es uns erlaubt, unser Wissen über einen Bereich zu nutzen, um über einen anderen Bereich nachzudenken. Es sei auch an die Idee erinnert, dass, weil ,Metapher' primär etwas Konzeptuelles sei (,a matter of thought“), ${ }^{8}$ unterschiedliche Medien die gleiche konzeptuelle Metapher manifestieren - weshalb die Art und Weise einer Artikulation für das Modell zweitrangig ist. Darüber hinaus ist für eine Verortung der KMT im Zwei-WeltenModell bezeichnend, dass sich die KMT für Sprache und Kommunikation interessiert, weil sie daran, wenn auch nicht grammatische Regeln, aber doch in gewisser Weise regelhafte konzeptuelle Schemata beschreiben möchte, „denen wir“, um wieder mit Krämer zu sprechen, „im Sprechen und Kommunizieren folgen“. ${ }^{9}$ Das automatische Verfügen über solche Schemata scheint als eine Art Erfahrungs-Wissen bestimmbar, welches (implizit oder explizit) in Sprechern oder eben in Filmemachern oder Filmzuschauern seine Repräsentation findet. Und schließlich ist mit Image-Schemata und Primärmetaphern auch eine für das ZweiWelten-Modell typische „Analytizität“ gegeben, d.h. die Idee, metaphorische Ausdrücke „in letzte Elementareinheiten zu zerlegen“. ${ }^{10}$

Entsprechend signifikant ist, dass von den vorgestellten Ansätzen denn auch so prominent auf ein System abgehoben wird, das der Kognition (im weitesten

6 Für diesen Hinweis danke ich Cornelia Müller.

7 Sybille Krämer: Zur Kinästhesie der verkörperten Sprache. In: Kunst der Bewegung. Kinästhetische Wahrnehmung und Probehandeln in virtuellen Welten. Hrsg. v. Christina Lechtermann und Carsten Morsch. Bern/Berlin: Peter Lang 2004, S. 343-356, hier S. 345. Angemerkt sei, dass gerade in den Gründungsschriften der KMT die Sprachbeispiele gänzlich aus raum-zeitlich spezifizierbaren Gebrauchskontexten herausgelöst sind.

8 Coëgnarts/Kravanja: Embodied Visual Meaning, S. 89.

9 Vgl. Krämer: Zur Kinästhesie der verkörperten Sprache, S. 344.

10 Ebd., S. 345. Mit „Analytizität“ ist hiermit einer von drei Aspekten herausgegriffen, die Krämer im Rahmen von „Diskursivität“ als dem dritten Merkmal des Zwei-Welten-Modells nennt. Krämer, die im Wesentlichen sprachtheoretisch argumentiert, zählt zur Diskursivität über die Analytizität hinaus noch Linearität und Arbitrarität (siehe ebd.). 
Sinne) zugrunde liegt. ${ }^{11}$ Durchgängig findet sich die Annahme, dass Filmemacher als Produzenten audiovisueller Bilder mehr oder weniger bewusst auf eine konzeptuelle System-Ebene Bezug nehmen, und in dieser intentionalen Bezugnahme die Ursache für die Wirkmächtigkeit wie auch Kommunikationsfähigkeit von Filmen liegt. Und obwohl sprachwissenschaftliche Metaphernkonzepte für diese Arbeiten vordergründig keine Rolle spielen, ${ }^{12}$ bleibt damit doch eine Reminiszenz an traditionelle Metapherntheorien bestehen. Denn die Metapher wird - klassischer Weise - als statisch gegebene Bedeutungseinheit entworfen: Eben weil der Bezug auf ein konzeptuelles System als Entität ${ }^{13}$ immer gegeben ist, kann produzentenseitig für die Produktion audiovisueller Metaphern über eine solche Bedeutungseinheit intentional verfügt und davon ausgegangen werden, dass sie - insbesondere bei den konzeptuellen Metaphern - rezipientenseitig automatisch abgerufen wird. Produktionsseitig wird also ein (relativ) ${ }^{14}$ konstruktives Moment in den Mittelpunkt gerückt, insofern sich Mediengestalter vor die Aufgabe gestellt sehen, Abstraktes darzustellen, und der Rekurs auf konzeptuelle Metaphern es ihnen ermöglicht, Abstraktes in eine anschauliche Form zu bringen. Rezeptionsseitig wiederum erscheint die Metapher eher als Automatismus. Denn ist der produktionsseitige Konstruktionsakt (d. h. die Entscheidung und Umsetzung, was wie zu inszenieren ist) erst einmal geleistet, so ist für den Zuschauer wiederum, der die Bilder konsumiert, die Metapher eine Gegebenheit, da über das gleiche, weitgehend statisch gedachte System verfügt wird. Der Aspekt einer Gegebenheit der Metapher ist auch für Forcevilles Ansatz zu kreativen Metaphern in Anschlag $\mathrm{zu}$ bringen, obwohl dort eine Zuschaueraktivität im Mittelpunkt steht - doch diese läuft, wie hier argumentiert wurde, letztlich auf das Re-Konstruieren eines Gegebenen hinaus. Infolgedessen aber bleibt bei KMT-orientierten Ansätzen denn auch ein dynamischer Charakter von Metaphern weitestgehend außen vor.

11 Auch das Genresystem (beruhend auf einem taxonomischen Genreverständnis) wird darin etwa stark gemacht. Vgl. hierzu z. B. Fahlenbrach: Audiovisuelle Metaphern.

12 Forcevilles Bezugnahme auf Blacks Interaktionstheorie der Metapher bildet hier eine Ausnahme.

13 Es ist insbesondere Lakoff, der ein solches Verständnis eines konzeptuellen Systems als Entität in der Kognitiven Linguistik stark macht. Vgl. z. B. Lakoff: The Neural Theory of Metaphor. Gibbs beispielsweise vertritt ein dynamisches Verständnis, wodurch noch das System selbst emergent ist. Vgl. Gibbs: Embodiment and Cognitive Science; Raymond W. Gibbs, Jr./Lynne Cameron: The Social Cognitive Dynamics of Metaphor Performance. In: Cognitive Systems Research 9.1-2 (2008), S. 64-75; Raymond W. Gibbs, Jr.: Metaphor Wars. Conceptual Metaphors in Human Life. Cambridge: Cambridge University Press 2017.

14 Das konstruktive Moment ist relativ, insofern ein Rekurs auf Vorgängiges im Zentrum steht. 
Gerade weil von der Metapher bzw. von metaphorischer Bedeutung als einer Gegebenheit ausgegangen wird, weil es als etwas entworfen ist, über das zu verfügen ist, bietet sich wiederum ein Sender-Empfänger-Modell von Kommunikation für KMT-orientierte Ansätze an. Damit geht einher, dass die Analysen ihren Ausgangspunkt mehr von konzeptuellen Metaphern (ob nun einfachen oder komplexen) bzw. von bestehenden Konzepten nehmen und weniger zuvorderst beim audiovisuellen Bild ansetzen (dies gilt auch für den Fall, wenn filmische Inszenierungsstrategien wie etwa eine Kamerabewegung argumentativ eingebunden werden). Verkörperung wird insofern auch wesentlich bezogen auf systemische, außerfilmische Muster von Erfahrung gedacht, und es wird dementsprechend nicht vom audiovisuellen Bild als einer konkreten Form von (Wahrnehmungs-) Erfahrung ausgegangen. Das in besonderer Weise Sinnlich-Bildliche von Filmen und die körperliche Wahrnehmung dessen in einem Sehen und Hören bleibt eine Leerstelle. 
